\title{
Aortic regurgitation and sinus of Valsalva-right atrial fistula after blunt thoracic trauma
}

\author{
ROGER B REHR, MICHAEL MACK, BRIAN G FIRTH \\ From the Departments of Internal Mediçine (Cardiology Division) and Surgery (Cardiothoracic Division), University \\ of Texas Health Science Center and Parkland Memorial Hospital, Dallas, Texas, USA
}

SUMMARY Non-penetrating chest trauma commonly causes a wide variety of cardiac injuries. Disruption of the aortic valve with resultant aortic regurgitation is not uncommon; conversely, a sinus of Valsalva-right atrial fistula, in the absence of a congenital sinus of Valsalva aneurysm, has been reported only once previously. This report describes the detection by preoperative cardiac catheterisation of both aortic regurgitation and a sinus of Valsalva-right atrial fistula after blunt chest trauma, and its surgical management. The need for preoperative cardiac catheterisation in patients suffering from non-penetrating cardiac trauma is emphasised, even when the diagnosis appears clear, because of the diverse nature and possible multiplicity of cardiac lesions.

Non-penetrating chest trauma commonly causes cardiac injury, and a wide variety of anatomical derangements may ensue. ${ }^{1}$ The most frequent valvular lesion described in those who survive the trauma has been disruption of the aortic valve with resultant aortic regurgitation. ${ }^{1} \mathrm{~A}$ much less common lesion resulting from blunt trauma is a tear in the aortic wall resulting in a sinus of Valsalva-right atrial fistula, with an associated left to right shunt. Only one such case has previously been reported. ${ }^{2}$

The following case report describes a patient who presented with both aortic regurgitation and a sinus of Valsalva-right atrial fistula after non-penetrating chest injury. The clinical signs of aortic valve disruption masked those of the sinus of Valsalva-right atrial fistula and the presence of the fistula was only detected by preoperative cardiac catheterisation.

\section{Case report}

A 28-year-old tall thin previously healthy white man was involved in a car accident. He sustained multiple contusions to his chest and extremities but no serious injuries were apparent. He was seen at an emergency room and discharged after his chest radiograph was reported to be normal. He felt well except for chest wall pain until two weeks after the accident. Over a three to four day period he then gradually developed progressive symptoms of pulmonary congestion which rapidly culminated in his admission to a local hospital. There he was found to be in severe pul- monary oedema with a murmur of aortic regurgitation. After a 5 litre diuresis in 24 hours his condition improved and he was transferred to Parkland Memorial Hospital.

On arrival he was very dyspnoeic while sitting at an angle of 60 degrees. Blood pressure was 130/30/0 $\mathrm{mmHg}$ in both arms and pulse rate was 100 a minute, with bounding peripheral and carotid pulses. He had a high arched palate but no ectopia lentis. There was no jugular venous distension but chest examination disclosed bilateral basal râles and dullness to percussion. The apex beat was hyperdynamic in the fifth intercostal space at the midclavicular line and there was a fourth heart sound. A grade $3 / 6$ systolic ejection murmur and a grade $3 / 6$ long early diastolic decrescendo murmur were heard, loudest along the left sternal border, and an Austin-Flint rumble was heard at the apical area. There was no continuous murmur. Abdominal examination was normal and there was no peripheral oedema.

Chest radiographs showed the heart size to be at the upper limits of normal with bilateral pleural effusions, pulmonary venous congestion, and prominent pulmonary arteries. The electrocardiogram showed sinus tachycardia but was otherwise normal. M-mode echocardiography showed fluttering of the anterior mitral valve leaflet as well as premature closure of the mitral valve. The left atrial and aortic root dimensions were both increased at $4.2 \mathrm{~cm}$, as were the left ventricular end-systolic and end-diastolic dimensions (4.3 and $6.9 \mathrm{~cm}$, respectively). There was no left ventricu- 
lar hypertrophy. Two dimensional echocardiography suggested a flail aortic valve leaflet. The tricuspid valve appeared normal both by $M$-mode and two dimensional echocardiography. A cut film aortogram was performed to rule out dissection of the aorta. This showed moderately severe aortic regurgitation but no other abnormality.

At cardiac catheterisation routine oximetry showed a significant step-up in oxygen saturation (Table). Though the saturation step-up appeared to be at the right ventricular level, the identical saturation measurements throughout the right ventricle raised the question of a more proximal shunt with streaming of blood through the upper part of the tricuspid valve. The calculated pulmonary blood flow using the Fick principle was $10 \mathrm{l} / \mathrm{min}$, systemic blood flow was $4.5 \mathrm{l} / \mathrm{min}$, yielding a calculated left to right shunt of $2 \cdot 2: 1$. Indicator dilution curves suggested a 1.4:1 left to right shunt and no right to left shunt. Haemodynamic measurements showed normal right sided pressures (right atrial mean pressure $5 \mathrm{mmHg}$, pulmonary arterial pressure $27 / 12 \mathrm{mmHg}$ ); mean pulmonary capillary wedge pressure was $14 \mathrm{mmHg}$, and left ventricular end-diastolic pressure was $27 \mathrm{mmHg}$. Cineangiography of the aortic root showed slight dilatation of the aortic annulus, severe aortic regurgitation, and passage of contrast from the right sinus of Valsalva into the right atrium (Fig.). Left ventricular angiography showed no mitral regurgitation, normal wall motion, and a left ventricular ejection fraction of 0.73 . The coronary arteries were angiographically normal.

At operation there was no evidence of a haemopericardium and the right sinus of Valsalva was found to bulge into the right atrium. A jet of blood from the right sinus of Valsalva could be palpated as it

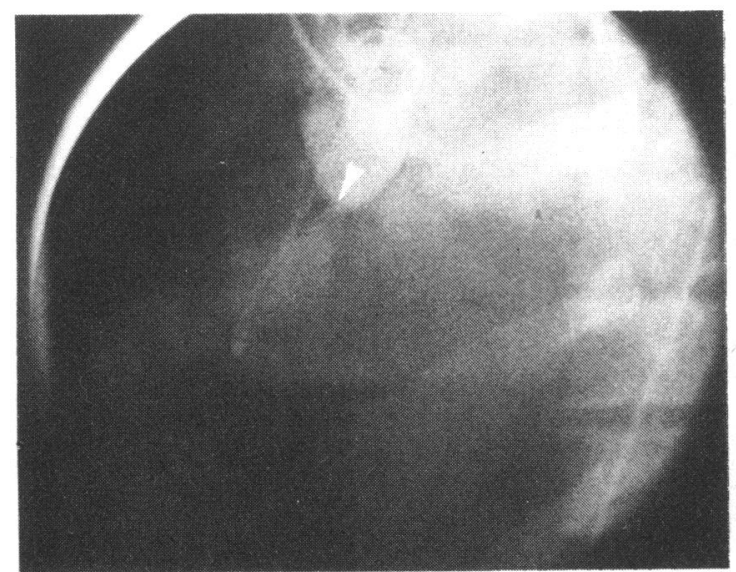

(A)

Fig. Supravalvular aortogram in the left anterior oblique projection. Contrast material passes from the sinus of Valsalva into the right atrium $(A)$ and into the left ventricle via the aortic valve $(B)$.
Table Oximetry measurements

\begin{tabular}{ll}
\hline & Oxygen saturation (\%) \\
\hline First set & 80 \\
Left pulmonary artery & 77 \\
Main pulmonary artery & 77 \\
Right pulmonary artery & 76 \\
Right ventricular outflow tract & 64 \\
Right atrium at tricuspid valve & 68 \\
High inferior vena cava & 54 \\
Low inferior vena cava & \\
Second set & 62 \\
High superior vena cava & 66 \\
Low superior vena cava & 69 \\
Superior vena cava at right atrium & 67 \\
High right atrium & 72 \\
Mid right atrium & 69 \\
Low right atrium & 65 \\
High inferior vena cava & 52 \\
Low inferior vena cava & \\
Third set & 79 \\
Main pulmonary artery & 78 \\
Right ventricular outflow tract & 78 \\
Right ventricle mid-cavity & 77 \\
Right ventricle at tricuspid valve & 79 \\
Right ventricular apex & 70 \\
Right atrium at tricuspid valve & 70 \\
High inferior vena cava & 70 \\
\hline
\end{tabular}

entered the right atrium above the tricuspid valve. The aortic valve was bicuspid. There was a one centimetre tear in the aortic valve across the rudimentary commissure of the right cusp, with associated aortic valvular regurgitation. The aortic opening of the sinus of Valsalva-right atrial fistula was situated in the aortic wall above the right cusp. The aortic valve was excised and replaced with a $29 \mathrm{~mm}$ CarpentierEdwards porcine heterograft and the fistula was closed by direct suture from the aortic side. A biopsy of the aortic wall was histologically normal. The patient had an uncomplicated postoperative course.

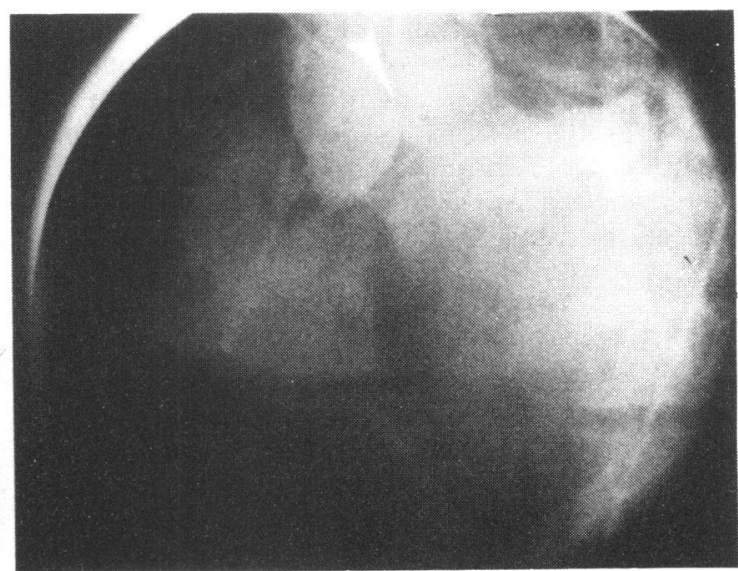

(B) 


\section{Discussion}

Aortic regurgitation resulting from blunt chest trauma has been described both as an isolated injury and as one of a constellation of injuries. ${ }^{13-5}$ Though rupture of congenital sinus of Valsalva aneurysms is well described, ${ }^{67}$ a sinus of Valsalva-right atrial fistula resulting from penetrating trauma is comparatively rare. ${ }^{89}$ The occurrence of such a fistula after nonpenetrating chest trauma is even less common: only one such case has been reported. ${ }^{2}$ This was not associated with a congenital sinus of Valsalva aneurysm.

In the absence of a congenital sinus of Valsalva aneurysm which impinges on the right atrium, there is normally a space between the right atrium and the aorta. Thus, an acute tear of the proximal aorta generally leads to a leak into the pericardial space rather than into the right atrium. Therefore, the pathogenesis of a sinus of Valsalva-right atrial fistula is likely to represent a subacute process: the tear in the aortic wall results in a limited dissection of the aorta with haematoma formation, which impinges on the right atrium and subsequently ruptures into that chamber. This delayed rupture into the right atrium may have been responsible for the late deterioration in our patient's condition, which allowed full evaluation and surgical treatment, and probably explains why acute haemopericardium and cardiac tamponade did not occur.

The combination of aortic valvular regurgitation and sinus of Valsalva-right atrial fistula with a left to right shunt has not previously been described. The physical findings of the aortic regurgitation (systolic and diastolic murmurs, wide pulse pressure, and bounding pulses) masked the features of a sinus of Valsalva-right atrial fistula. Furthermore, though echocardiography suggested a torn aortic leaflet and aortic regurgitation, it did not show diastolic fluttering of the tricuspid valve (as described previously with a similar fistula ${ }^{2}$ ), or any protrusion of the right sinus of Valsalva. Since the severity of the patient's aortic valvular regurgitation appeared to explain his clinical status adequately, we did not suspect the presence of the fistula before cardiac catheterisation, and would not have detected the fistula before, or possibly even at the time of operation, had catheterisation been omitted.

This case report serves to emphasise the need for preoperative cardiac catheterisation in patients suffering from non-penetrating cardiac trauma, even when the diagnosis appears clear and the patients's condition stable, because of the diverse nature and the possible multiplicity of cardiac lesions which may not be detected by non-invasive techniques.

We thank Drs Roy V Markham, Robert E Rude, and Melvin Platt for their assistance in the preparation of this report and Ms Sarah Hawkins and Nancy Smith and Mr Randy Christian for technical assistance.

\section{References}

1 Parmley LF, Manion WC, Mattingly TW. Nonpenetrating traumatic injury of the heart. Circulation 1958; 18: $371-96$.

2 DeSa'Neto A, Padnick MB, Desser KB, Steinhoff NG. Right sinus of Valsalva-right atrial fistula secondary to nonpenetrating chest trauma. Circulation 1979; 60: 205-9.

3 Beall AC Jr, Shirkey AL. Successful surgical correction of traumatic aortic valve regurgitation. $\mathcal{F} A M A$ 1964; 187: 507-10.

4 Leonard JJ, Harvey WP, Hufnagel CA. Rupture of the aortic valve: a therapeutic approach. $N$ Engl f Med 1955; 252: 208-12.

5 Levine RJ, Roberts WC, Morrow AG. Traumatic aortic regurgitation. Am f Cardiol 1962; 10: 752-63.

6 Boutefeu JM, Moret PR, Hahn C, Hauf E. Aneurysms of the sinus of Valsalva. Am $\mathcal{F}$ Med 1978; 65: 18-24.

7 Kieffer SA, Winchell P. Congenital aneurysms of the aortic sinuses with cardioaortic fistula. Diseases of the Chest 1960; 38: 79-96.

8 Berger M, Bhalla R, Jelveh M, Goldberg E. Continuous murmur following chest trauma. Arch Intern Med 1979; 139: $1305-6$.

9 Morris GC Jr, Foster RP, Dunn JR, Cooley DA. Traumatic aortico-ventricular fistulas. Report of two cases successfully repaired. Am Surg 1958; 24: 883-8.

Requests for reprints to Dr Brian G Firth, Ischemic Heart Center, L5 134, University of Texas Health Science Center at Dallas, 5323 Harry Hines Boulevard, Dallas, Texas 75235, USA. 